\title{
Clinical Investigation of Matrix Metalloproteinases, Tissue Inhibitors of Matrix Metalloproteinases, and Matrix Metalloproteinase/Tissue Inhibitors of Matrix Metalloproteinase Complexes and Their Networks in Apical Periodontitis
}

\author{
Frederico C. Martinbo, DDS, MSc, PbD, * Flávia F.C. Teixeira, DDS, MSc, * \\ Flávia G.R. Cardoso, DDS, MSc, ${ }^{{ }^{\dagger}}$ Nádia S. Ferreira, DDS, MSc, PbD, ${ }^{\ddagger}$ \\ Gustavo G. Nascimento, DDS, MSc, PbD, ${ }^{\neq}$Cláudio A.T. Carvalbo, DDS, MSc, PhD, * \\ and Márcia C. Valera, DDS, MSc, PbD*
}

\section{Ahstract}

Introduction: This clinical study investigated the levels of metalloproteinases (MMPs) and their tissue inhibitors (TIMPs) and respective forms (MMP/TIMP complexes) in apical periodontitis to determine their networks in the development of clinical/radiographic features, thus quantifying the levels of endotoxins (lipopolysaccharides) present in primarily infected root canals with apical periodontitis. Methods: Twenty primarily infected root canals with apical periodontitis were selected. The presence of pain on palpation, tenderness to percussion, and the size of the radiographic lesion were recorded. The levels of MMPs (MMP-1, -2 , and -9), TIMPs (TIMP-1 and -2), and their MMP/TIMP complexes (MMP1/TIMP-1, MMP-1/TIMP-2, MMP-2/TIMP-1, MMP-2/ TIMP-2, MMP9/TIMP-1, and MMP-9/TIMP-2) present in the periapical interstitial fluid were measured using the enzyme-linked immunosorbent assay. The kinetic chromogenic LAL test was used to quantify endotoxins. Results: A higher mean level of MMP-9 (968.35 \pm $342.00 \mathrm{pg} / \mathrm{mL}$ ) was followed by MMP-2 (894.00 \pm $591.62 \mathrm{pg} / \mathrm{mL})$ and MMP-1 (789.43 $\pm 342.83 \mathrm{pg} / \mathrm{mL})$. The linear regression analysis revealed a positive association of MMP-1 with both MMP-2 and MMP-9 (all $P<.001)$. TIMP-1 $(481.79 \pm 86.09 \mathrm{pg} / \mathrm{mL})(24 / 24)$ was found in higher levels than TIMP-2 (206.45 $\pm 86.09 \mathrm{pg} / \mathrm{mL})(P<.05)$, including a positive correlation of MMP-1 with both TIMP-1 and TIMP-2 (all $P<.05)$. Higher mean levels of MMP1, -2 , and -9

were found in teeth with larger-size radiolucent lesions $(>7 \mathrm{~mm}$ ) compared with smaller ones $(\leq 7 \mathrm{~mm}$ ) (all $P<.01)$. Higher levels of MMP-1 decreased the chance of TTP, whereas MMP-9 (odds ratio $=0.97$ ) increased the chance of pain on percussion (odds ratio $=1.01$ ). Higher levels of endotoxins present in root canals were positively correlated with larger amounts of MMP $-9(P<.05)$. Conclusions: MMPs, TIMPs, and their complexes (MMP/TIMP) are involved in apical periodontitis by interacting with complex networks in the development of clinical features and the severity of bone destruction. (J Endod 2016;42:1082-1088)

\section{Key Words}

Endodontics, endotoxin, infection, root canal

$\Lambda$ pical periodontitis is Aa tissue breakdown established in the periapical tissue provoked by the interplay between bacterial infection of the root canal systems and the local immune response (1). Such a local immune response is very complex and involves degradation of the extracellular matrix (ECM) components, the main components of connective tissue (2).

Matrix metalloproteinases (MMPs) (3), a family of zinc- and calcium-dependent endopeptidases, are strongly associated with the breakdown of ECM (4) and play a major role in bone remodeling and resorption (5). According to their target protein, MMPs are divided into several families (6) as follows: collagenase (MMP-1 (7)), gelatinases (MMP-2 (8)), stromelysin (MMP-3 (7)), and matrixin (MMP-9 (9)). Different subtypes of MMPs, including MMP-1, $-2,-3,-7,-8$, and -9 , have been detected in apical periodontitis $(3,10-16)$. Of a clinical concern, elevated MMP levels have been correlated with nonhealing lesions $(17,18)$.

From the *Department of Restorative Dentistry, Endodontic Division, São Jose dos Campos Dental School, State University of São Paulo, UNESP; ${ }^{\dagger}$ Department of Odontology, Endodontic Division, University of Taubaté, Taubaté, São Paulo, São Paulo; and Department of Semiology, Federal University of Pelotas, School of Dentistry, Pelotas, Rio Grande do Sul, Brazil.

Address requests for reprints to Dr Frederico C. Martinho, São José dos Campos Dental School, State University of São Paulo-UNESP, Department of Restorative Dentistry, Endodontic Division, Eng Francisco José Longo, 777, São José dos Campos, SP, Brazil, CEP 12245-000. E-mail address: Frederico.martinho@fosjc.unesp.br 0099-2399/\$ - see front matter

Copyright (C) 2016 American Association of Endodontists. http://dx.doi.org/10.1016/j.joen.2016.04.001 
The zinc-dependent endopeptidase activities of the MMPs are inhibited specifically by their intrinsic inhibitors called tissue inhibitors of MMPs (TIMPs) (19-21). TIMPs have been detected in apical periodontitis (16, 22), with TIMP-1 and -2 being multifunction proteins (23). Particularly, TIMP-1 has been suggested as an enhancer or inhibitor of bone resorption depending on whether TIMP-1 concentrations are low or high, respectively (24). Despite the biological activities of MMPs, independent of the MMP-inhibitory activity, the imbalance between the levels of MMPs and TIMPs is critical for ECM remodeling being able to provoke tissue destruction (23). Thus, TIMPs can bind tightly to the active proteinases (MMPs) to form the MMP/TIMP complex (25-27). The MMP/TIMP complex has been speculated by other research fields as a potential biological biomarker for the possible prediction of changes in the course of diseases $(28-30)$.

To help understand the participation of MMPs and TIMPs and their network in apical periodontitis, this clinical study sought to investigate the levels of MMPs and TIMPs and their respective forms (MMP/TIMP complexes) by determining their networks in the development of clinical/radiographic features, thus quantifying the levels of endotoxins (lipopolysaccharides [LPSs]) present in primarily infected root canals with apical periodontitis.

\section{Patient Selection}

\section{Materials and Methods}

Twenty patients attending São José dos Campos Dental School, São José dos Campos, São Paulo, Brazil, for primary endodontic treatment were included in the present study. The age of patients ranged from 22-45 years old (mean $=35$ years, 7 women and 13 men). A detailed dental and general history was obtained from each patient. Those who had received antibiotic treatment during the past 3 months and/or who had any systemic health disorder that might affect the inflammatory response were excluded.

The Human Research Ethics Committee of the São José dos Campos Dental School approved the protocol describing the sample collection for this investigation, and all the volunteer patients signed an informed consent form.

All the single-rooted teeth were maxillary teeth with primary endodontic infection showing the presence of 1 root canal and the absence of periodontal pockets deeper than $4 \mathrm{~mm}$. Teeth that could not be isolated with a rubber dam were also excluded. None of them reported any type of systemic disease. The following clinical/radiographic features were recorded: clinical symptomatology (spontaneous pain) $(11 / 20)$, pain on palpation $(9 / 20)$, tenderness to percussion $(7 / 20)$, and size of radiolucent area $>7 \mathrm{~mm}(11 / 20)$.

\section{Sampling Procedures}

The files, instruments, and all materials used in this study were treated with $\mathrm{Co}^{60}$ gamma radiation ( $20 \mathrm{kGy}$ for 6 hours) for sterilization and elimination of pre-existing endotoxins (EMBRARAD; Empresa Brasileira de Radiação, Cotia, SP, Brazil) (31). The method used for disinfection of the operative field has been previously described elsewhere (32). Briefly, the teeth were isolated with a rubber dam. The crown and surrounding structures were disinfected with $30 \% \mathrm{H}_{2} \mathrm{O}_{2}$ (volume/volume) for 30 seconds followed by $2.5 \%$ sodium hypochlorite $(\mathrm{NaOCl})$ for the same period of time and then inactivated with $5 \%$ sodium thiosulfate. The sterility of the external surfaces of the crown was checked by taking a swab sample from the crown surface and streaking it onto blood agar plates, which were then incubated both aerobically and anaerobically.
A 2-stage access cavity preparation was made without the use of water spray but under manual irrigation with sterile/apyrogenic saline solution and by using a sterile/apyrogenic high-speed diamond bur. The first stage was performed to promote a major removal of contaminants, including carious lesion and restoration. In the second stage, before entering the pulp chamber, the access cavity was disinfected according to the protocol described previously. Sterility of the internal surface of the access cavity was checked as previously described, and all procedures were performed aseptically. An endotoxin sample was taken by introducing sterile/apyrogenic paper points (size \#15; Dentsply Maillefer, Ballaigues, Switzerland) into the full length of the canal determined radiographically and retained in position for 60 seconds for sampling. Immediately after, the sample was placed in pyrogen-free glass and immediately suspended in $1 \mathrm{~mL}$ limulus amebocyte lysate water and frozen at $-80^{\circ} \mathrm{C}$ for further dosage of endotoxins using a kinetic chromogenic limulus amebocyte lysate assay (Lonza, Walkersville, MD).

After endotoxin sampling, the root canal length was determined from the preoperative radiograph and confirmed using an apex locator (RomiApex A-15; Romidan Dental Solution, Kiryat-Ono, Israel). The root canals were prepared by using Mtwo files (VDW, Munich, Germany) according to the manufacturer's instructions. The files were adapted to an electric motor (VDW), and all instruments were used within the working length in a gentle in-and-out motion. The instrumentation sequence was as follows: 0.04 taper size \#10 instrument, 0.05 taper size \#15 instrument, 0.06 taper size \#20 instrument, 0.06 taper size \#25 instrument, 0.05 taper size \#30 instrument, 0.04 taper size \#35 instrument, 0.04 taper size \#40 instrument, and 0.07 taper size \#25 instrument. The use of each instrument was followed by irrigation with disposable syringes and 30-G NaviTip needles (Ultradent, South Jordan, UT) using $5 \mathrm{~mL} 2.5 \% \mathrm{NaOCl}$ solution. Before the second sampling after instrumentation, $\mathrm{NaOCl}$ was inactivated with $5 \mathrm{~mL}$ sterile $0.5 \%$ sodium thiosulfate during a 1-minute period, which was then removed with $5 \mathrm{~mL}$ sterile/apyrogenic water. Next, the second bacterial sampling was performed as previously described.

After root canal instrumentation, the samples were collected from the interstitial fluid in the apical periodontitis according to Martinho et al (31). Three sterile paper points were introduced into the root canal through the root apex $(2 \mathrm{~mm})$ and kept there for 1 minute for sampling inflammatory contents from the interstitial fluid in the apical tissues. After withdrawal, the paper points were cut $4 \mathrm{~mm}$ from the tip, dropped into a 1.5-mL sterile plastic tube, and immediately stored at $-80^{\circ} \mathrm{C}$ for further analysis of the cytokines.

\section{Quantification of MMPS, TIMPs, and MMP/TIMP Complexes Quantification of Inflammatory Cytokines [Enzyme- linked Immunosorbent Assay]. The amount of MMPs} (MMP-1 [Cat \#DY901], -2 [Cat\#DY902], and -9 [Cat \#DY911]), TIMPs (TIMP-1 [Cat\# DY970] and -2 [Cat\#DY971]), and MMP/TIMP complexes (MMP-1/TIMP-1 [Cat\# DY1550], MMP-1/TIMP-2 [Cat \#DY1553], MMP-2/TIMP-1 [Cat \#DY1496], MMP-2/TIMP-2 [Cat \#DY1497], MMP-9/TIMP-1 [Cat \#DY1449], and MMP-9/TIMP-2 [Cat\#DY1453]) sampled from the interstitial fluid in the apical tissues were measured using an enzyme-linked immunosorbent assay (ELISA) kit (R\&D Systems, Inc, Minneapolis, MN). Next, standard and sample solutions were added to the ELISA well plate, which had been precoated with specific monoclonal capture antibody for MMP-1, MMP-2, MMP-3, TIMP-1, TIMP-2, MMP-1/TIMP-1, MMP-1/TIMP-2, MMP-2/TIMP-1, MMP-2/TIMP-2, MMP-9/TIMP-1, and MMP-9/TIMP-2. After it was gently shaken for 3 hours at room temperature, the polyclonal antiMMP-1, -MMP-2, -MMP-3, -TIMP-1, -TIMP-2, -MMP-1/TIMP-1, 
-MMP-1/TIMP-2,-MMP-2/TIMP-1, -MMP-2/TIMP-2, -MMP-9/TIMP1, and-MMP-9/TIMP-2 antibodies conjugated with horseradish peroxidase were added to the solution and incubated for 1 hour at room temperature. A substrate solution containing hydrogen peroxidase and chromogen was added and allowed to react for 20 minutes. The levels of MMPs, TIMPs, and MMP/TIMP complexes were assessed with a micro-ELISA reader at $450 \mathrm{~nm}$ and normalized with standard solution. Each densitometric value, expressed as mean \pm standard deviation, was obtained from 3 independent experiments.

\section{Quantification of Endotoxins}

\section{Quantification of Endotoxins [LPSs] by Kinetic Chromo-} genic Limulus Amebocyte Lysate Assay. The kinetic chromogenic limulus amebocyte lysate assay (Lonza) was used for quantification of endotoxins. Escherichia coli endotoxin was used as the standard. A positive control (root canal sample contaminated with a known amount of endotoxin) was included for each sample to determine the presence or absence of interfering agents. For the test, $100 \mu \mathrm{L}$ apyrogenic water (reaction blank), 5 standard endotoxin solutions $(0.005-50$ endotoxin units $[\mathrm{EU}] / \mathrm{mL})$, root canal samples, and positive controls (each root canal sample contaminated with a known concentration of endotoxin $[10 \mathrm{EU} / \mathrm{mL}]$ ) were added to a 96 -well apyrogenic plate. The tests were performed in quadruplicate. The plate was incubated at $37^{\circ} \mathrm{C} \pm 1^{\circ} \mathrm{C}$ for 10 minutes in a kinetic-QCL reader, which was coupled to a microcomputer by means of the WinKQCL software. Next, $100 \mu \mathrm{L}$ chromogenic reagent was added to each well. After the beginning of the kinetic test, the software continuously monitored absorbance at $405 \mathrm{~nm}$ in each microplate well and automatically calculated the $\log / \log$ linear correlation between the reaction time of each standard solution and the corresponding endotoxin concentration.

\section{Statistical Analysis}

Data collected for each case were tabulated in duplicate into a spreadsheet and statistically analyzed using STATA 12.0 software (StataCorp, College Station, TX). Initially, a descriptive analysis using the
Bartlett test was performed in order to explore data distribution. Data presenting non-normal distribution were appropriately transformed. Next, 1-way analyses of variances were conducted to determine the concentrations of MMPs, TIMPs, and MMP/TIMP complexes according to clinical and radiographic features. The Pearson correlation test was performed to correlate the levels of MMPs, TIMPs, and the MMP/TIMP complex. Linear regression was used to determine the possible associations of MMPs, TIMPs, and their complex (MMP/TIMP) with the size of the radiolucent area $(\mathrm{mm})$. Multivariate logistic regression models were performed to test associations between clinical features and MMPs. For variable selection in the multivariate logistic regression, the stepwise backward approach was used. Variables with a $P$ value $<.20$ in the crude analysis were included in the adjusted model as confounders. The significance level was set as $5 \%$ in the final model after adjustment.

\section{Results}

\section{Quantification of MMPs [MMP-1, -2, and -9], TIMPs [TIMP-1 and-2], and Complexes [MMPs/TIMPs] and Endotoxin}

MMP-1, -2, and -9 were detected in 100\% (20/20) of the samples. Higher mean levels of MMP-9 $(968.35 \pm 342.00 \mathrm{pg} / \mathrm{mL})$ followed by MMP-2 (894.00 $\pm 591.62 \mathrm{pg} / \mathrm{mL})$ and MMP-1 (789.43 \pm 342.83 $\mathrm{pg} / \mathrm{mL}$ ) were recorded. The linear regression analysis revealed a positive association of MMP-1 with MMP-2 (beta $=0.56, R^{2}=0.952$, $P<.001)$ and MMP-9 (beta $\left.=0.57, R^{2}=0.889, P<.001\right)$. Higher mean levels of TIMP-1 (481.79 \pm 86.09$)(20 / 20)$ were found compared with TIMP-2 (206.45 $\pm 86.09 \mathrm{pg} / \mathrm{mL}) \quad(20 / 20)$ $(P<.05)$. The linear regression analysis revealed positive associations of MMP-1 with TIMP-1 (beta $\left.=0.78, R^{2}=0.212, P<.05\right)$ and TIMP-2 (beta $\left.=1.83, R^{2}=0.231, P<.05\right)$. All MMP/TIMP complexes (MMP-1/ TIMP1，MMP-1/TIMP-2，MMP-2/TIMP-1，MMP-2/TIMP-2, MMP-9/ TIMP-1, and MMP-9/TIMP-2) were detected in $100 \%$ of the samples (20/20). Individual mean values of the MMP/TIMP complex are shown in Figure 1. MMP-2/TIMP-2 (394.54 $\pm 266.29 \mathrm{pg} / \mathrm{mL})$ and MMP-9/ TIMP-1 $(546.56 \pm 388.04 \mathrm{pg} / \mathrm{mL})$ were encountered in higher levels compared with all other complexes tested. Endotoxins were detected in

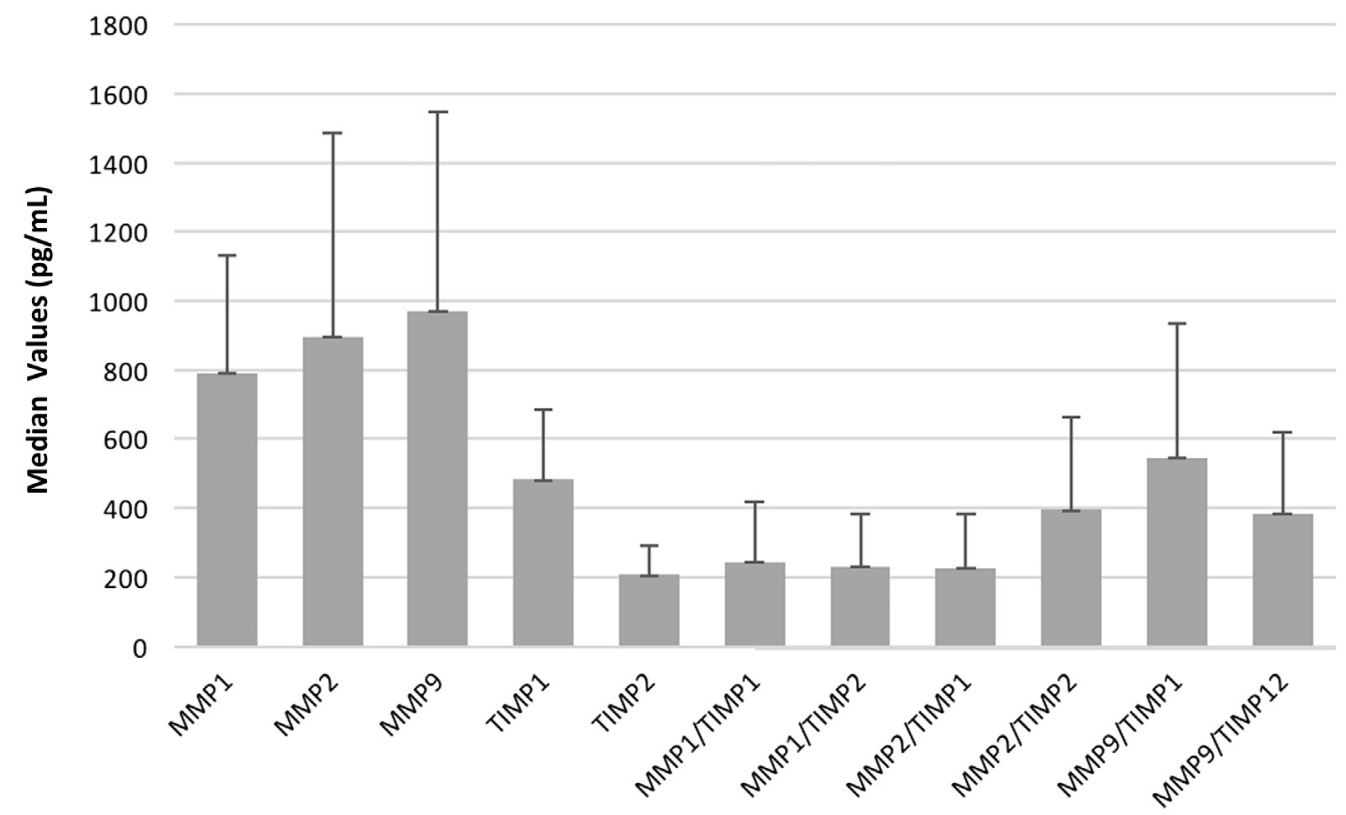

MMPs, TIMPs, and MMP/TIMP Complex

Figure 1. Distribution of the median values ( $\mathrm{pg} / \mathrm{mL}$ ) of MMPs, TIMPs, and the MMP/TIMP complex encountered in apical periodontitis. 
TABLE 1. Concentration of MMP, TIMP, and the MMP/TIMP Complex According to Clinical Symptoms and Radiographic Findings

\begin{tabular}{|c|c|c|c|c|c|c|c|c|c|}
\hline & \multicolumn{3}{|c|}{ Lesion size (mm) } & \multicolumn{3}{|c|}{ POP } & \multicolumn{3}{|c|}{ Symptom } \\
\hline & $<7$ & $>7$ & $P$ value & Absence & Presence & $P$ value & Absence & Presence & $P$ value \\
\hline MMP-1 & 536.99 (239.84) & $1041.88(221.33)$ & $<.0001$ & 718.55 (333.10) & $1072.99(235.82)$ & .060 & 719.73 (275.41) & $893.98(423.10)$ & .277 \\
\hline MMP-2 & $430.57(260.46)$ & 1356.75 (441.03) & $<.0001$ & $773.22(565.67)$ & 1375.43 (479.57) & .060 & $735.66(480.60)$ & $1030.66(692.90)$ & .148 \\
\hline MMP-9 & 547.04 (241.68) & $1389.66(505.59)$ & $<.0001$ & $827.77(512.60)$ & $1530.65(533.58)$ & .020 & 763.53 (364.97) & 1275.57 (722.35) & .049 \\
\hline Total MMP & $1513.8(739.58)$ & 3788.24 (1138.53) & $<.0001$ & 2319.05 (1390.27) & $3978.92(1244.41)$ & .043 & 2218.18 (1099.92) & 3300.27 (1833.27) & .114 \\
\hline TIMP-1 & 521.79 (252.97) & 441.70 (132.82) & .387 & $456.15(231.83)$ & $584.14(97.47)$ & .265 & 478.82 (228.94) & $486.13(164.74)$ & .939 \\
\hline TIMP-2 & $223.62(108.41)$ & $189.30(56.92)$ & .382 & 195.49 (91.64) & 250.34 (41.77) & .256 & $205.21(98.12)$ & $208.34(70.60)$ & .929 \\
\hline Total TIMP & 745.42 (361.39) & 631.01 (189.74) & .392 & 651.64 (305.48) & 834.49 (139.25) & .255 & 684.04 (327.06) & 694.48 (235.35) & .932 \\
\hline MMP-1/TIMP-1 & $413.21(14.97)$ & $75.28(7.35)$ & $<.0001$ & 264.65 (172.82) & $162.64(175.31)$ & .306 & 273.39 (176.68) & 200.53 (170.98) & .372 \\
\hline MMP-1/TIMP-2 & $377.32(18.88)$ & $84.42(10.21)$ & $<.0001$ & $249.80(149.68)$ & 155.18 (150.99) & .273 & $258.41(154.81)$ & 189.55 (144.77) & .331 \\
\hline MMP-2/TIMP-1 & 376.11 (32.84) & $72.71(7.81)$ & $<.0001$ & 241.38 (155.97) & $156.53(165.88)$ & .348 & $244.82(156.10)$ & 193.79 (164.72) & .492 \\
\hline MMP-2/TIMP-2 & 641.77 (116.59) & $147.52(20.01)$ & $<.0001$ & $424.03(262.94)$ & $277.10(283.58)$ & .337 & $419.33(247.84)$ & 357.62 (305.52) & .624 \\
\hline MMP-9/TIMP-1 & $904.19(47.73)$ & 150.63 (11.83) & $<.0001$ & 576.66 (388.58) & 330.38 (366.08) & .267 & 585.45 (380.98) & 440.35 (407.66) & .427 \\
\hline \multirow[t]{3}{*}{ MMP-9/TIMP-2 } & $611.06(19.87)$ & $155.76(15.42)$ & $<.0001$ & $411.86(235.60)$ & $269.61(219.67)$ & .289 & $418.10(235.80)$ & $331.39(237.40)$ & .432 \\
\hline & \multicolumn{5}{|c|}{ Previous Pain } & \multicolumn{4}{|c|}{ TTP } \\
\hline & \multicolumn{2}{|c|}{ Absence } & \multicolumn{2}{|c|}{ Presence } & $P$ value & Absence & \multicolumn{2}{|c|}{ Presence } & $P$ value \\
\hline MMP-1 & \multicolumn{2}{|c|}{$838.59(265.50)$} & \multicolumn{2}{|c|}{641.97 (524.76) } & .278 & \multirow{2}{*}{$\begin{array}{l}779.79(290.80) \\
843.85(522.06)\end{array}$} & \multicolumn{2}{|c|}{$818.36(510.86)$} & .834 \\
\hline MMP-2 & \multicolumn{2}{|c|}{$\begin{array}{l}951.74(527.07) \\
997.23(49968)\end{array}$} & \multicolumn{2}{|c|}{719.40 (799.77) } & .462 & & \multicolumn{2}{|c|}{$1043.09(819.41)$} & .529 \\
\hline MMP-9 & \multirow{2}{*}{\multicolumn{2}{|c|}{$\begin{array}{c}997.23(499.68) \\
2767.66(1270.38)\end{array}$}} & \multicolumn{2}{|c|}{$879.63(840.68)$} & .703 & $893.93(476.63)$ & \multicolumn{2}{|c|}{1191.61 (846.09) } & .330 \\
\hline Total MMP & & & \multirow{2}{*}{\multicolumn{2}{|c|}{$\begin{array}{r}2241.09 \text { (2164.49) } \\
355.62(166.83)\end{array}$}} & .493 & 2516.94 (1266.46) & 3053.2 & (2174.98) & .502 \\
\hline TIMP-1 & \multicolumn{2}{|c|}{$523.79(198.10)$} & & & .106 & $501.70(213.05)$ & $421 . \varepsilon$ & (163.88) & .456 \\
\hline TIMP-2 & 224.48 & $34.90)$ & 152.41 & 50) & .109 & $215.01(91.31)$ & $180 . \varepsilon$ & (70.24) & .557 \\
\hline Total TIMP & 748.28 & 283.00) & $508.02(2$ & $3.32)$ & .205 & 716.22 (304.36) & 602. & (234.11) & .478 \\
\hline MMP-1/TIMP-1 & 235.63 & 178.35) & 270.09 & $5.86)$ & .712 & $257.22(177.40)$ & 205. & (175.25) & .577 \\
\hline MMP-1/TIMP-2 & 222.14 & 153.77) & 257.06 & $5.10)$ & .664 & $242.62(155.82)$ & 195. & (145.68) & .561 \\
\hline MMP-2/TIMP-1 & 210.45 & 156.10) & 266.29 & 1.63) & .507 & $231.93(158.96)$ & 201. & (168.33) & .722 \\
\hline MMP-2/TIMP-2 & 360.54 & 246.99) & $496.95(3$ & 5.39) & .334 & $400.62(254.88)$ & $372 . \varepsilon$ & (330.05) & .867 \\
\hline MMP-9/TIMP-1 & 492.71 & 376.16) & $631.49(4$ & $9.41)$ & .503 & $547.81(380.78)$ & 466. & (488.90) & .695 \\
\hline MMP-9/TIMP-2 & 367.42 & $236.85)$ & 431.40 & $5.68)$ & .610 & $395.48(234.81)$ & 347. & (255.69) & .700 \\
\hline
\end{tabular}

MMP, matrix metalloproteinase; POP, pain on percussion; TIMP, tissue inhibitor of MMP.

Bold numbers signify $P<.05$ (statistical significance) 


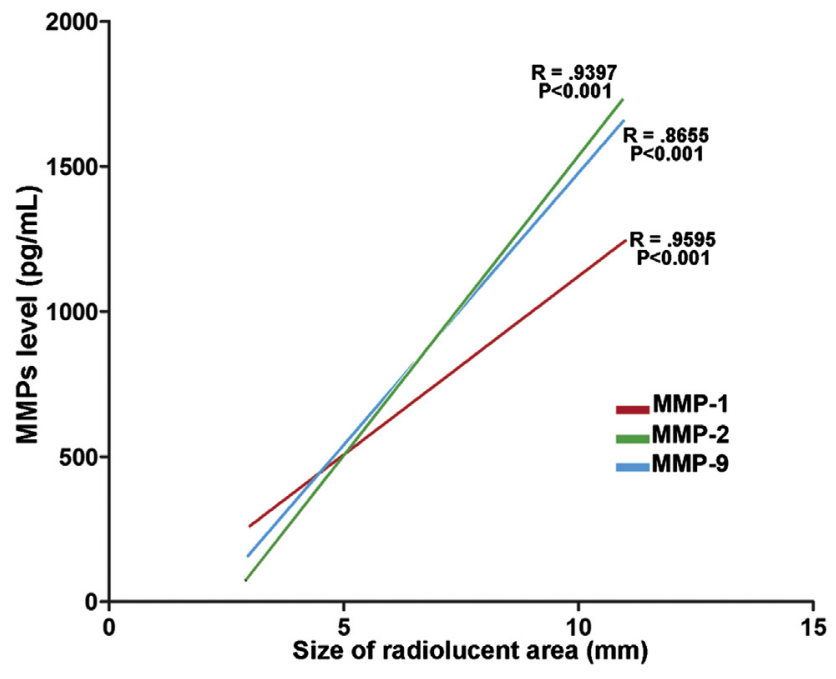

Figure 2. The Pearson correlation between MMP-1, -2 , and -9 and the size of the radiolucent area.

$100 \%$ of the root canal samples $(20 / 20)$, with mean values of 10.92 $\mathrm{EU} / \mathrm{mL}(1.75-128 \mathrm{EU} / \mathrm{mL})$. Higher levels of endotoxins present in root canals were positively correlated with larger amounts of MMP-9 $(P<.05)$.

\section{MMPs Versus Clinical/Radiographic Features}

Higher mean levels of MMP-1 $(1041.88 \pm 221.33 \mathrm{pg} / \mathrm{mL}$ vs $536.99 \pm 239.84 \mathrm{pg} / \mathrm{mL}, P<.001)$, MMP-2 $(1356.75 \pm 441.03$ $\mathrm{pg} / \mathrm{mL}$ vs $430.57 \pm 260.46 \mathrm{pg} / \mathrm{mL}, P<.001)$, and MMP-9 (1389.66 $\pm 505.59 \mathrm{pg} / \mathrm{mL}$ vs $547.04 \pm 241.68 \mathrm{pg} / \mathrm{mL}, P<.001)$ were found in teeth with larger-size radiolucent lesions $(>7 \mathrm{~mm})$ compared with smaller ones $(\leq 7 \mathrm{~mm})$, respectively (Table 1$)$. A positive correlation between higher levels of MMP-1, -2, and -9 and a larger area of bone destruction (Fig. 2) was also observed. Higher levels of MMP-2

TABLE 2. Multivariate Logistic Regression Models Performed to Test Associations between Clinical Features and Matrix Metalloproteinase (MMP)

\begin{tabular}{|c|c|c|c|c|}
\hline \multirow[b]{2}{*}{ Clinical features } & \multirow[b]{2}{*}{$\begin{array}{l}\text { Adjusted } \\
\text { odds ratio }\end{array}$} & \multicolumn{2}{|c|}{$\begin{array}{c}95 \% \\
\text { confidence } \\
\text { interval }\end{array}$} & \multirow[b]{2}{*}{$P$ value } \\
\hline & & $\begin{array}{l}\text { Lower } \\
\text { limit }\end{array}$ & $\begin{array}{l}\text { Upper } \\
\text { limit }\end{array}$ & \\
\hline \multicolumn{5}{|l|}{ Pain* } \\
\hline MMP-1 & 0.98 & 0.96 & 0.99 & 0.046 \\
\hline MMP-2 & - & - & - & - \\
\hline MMP-9 & 1.01 & 1.00 & 1.03 & 0.049 \\
\hline \multicolumn{5}{|c|}{ Tenderness to percussion ${ }^{\dagger}$} \\
\hline MMP-1 & 0.97 & 0.95 & 0.99 & 0.047 \\
\hline MMP-2 & 1.01 & 1.00 & 1.04 & 0.050 \\
\hline MMP-9 & - & - & - & - \\
\hline \multicolumn{5}{|l|}{ Pain on palpation ${ }^{\ddagger}$} \\
\hline MMP-1 & - & - & - & - \\
\hline MMP-2 & - & - & - & - \\
\hline MMP-9 & 1.01 & 1.00 & 1.02 & 0.040 \\
\hline \multicolumn{5}{|l|}{ Any symptom ${ }^{\ddagger}$} \\
\hline MMP-1 & 0.98 & 0.95 & 0.99 & 0.037 \\
\hline MMP-2 & - & - & - & - \\
\hline MMP-9 & 1.03 & 1.01 & 1.05 & 0.046 \\
\hline
\end{tabular}

*Adjusted for tissue inhibitor of matrix metalloproteinase (TIMP)-2.

${ }^{\dagger}$ Adjusted for TIMP-1, TIMP-2, and interleukin (IL)- $1 \beta$

${ }^{\ddagger}$ Adjusted for IL-6. decreased the odds of clinical symptomatology (odds ratio $[\mathrm{OR}]=$ $0.98)$, whereas higher levels of MMP- 9 increased this chance $(\mathrm{OR}=$ 1.01) (Table 2). In relation to tenderness to percussion, higher levels of MMP-1 reduced by $3 \%$ the chance of this outcome $(\mathrm{OR}=0.97)$, whereas higher levels of MMP-2 increased by $1 \%(\mathrm{OR}=1.01)$. Thus, higher levels of MMP-9 increased the chance of pain on palpation $(\mathrm{OR}=1.01)$ (Table 2).

\section{Discussion}

The data obtained in the present study revealed that MMPs, TIMPs, and their complexes (MMP/TIMP) are involved in apical periodontitis because they interact with complex networks in the development of clinical features and the severity of bone destruction.

All apical periodontitis samples were found to be positive for MMP-1, -2, and -9, which supports their role in the establishment/ development of the periapical inflammatory process $(3,11,14-16)$. Higher mean levels of MMP-9 and MMP-2 were recorded. Increased levels of MMP-2 and -9 have been reported in teeth with apical periodontitis and in wall extracts from jaw cysts $(3,10)$. Both gelatinases, gelatinase A (MMP-2) and B (MMP-9), are synthesized by both fibroblasts and pulp cells, and their presence favors the degradation of type IV collagens/gelatins (main components of the basement membrane) as well as denatured gelatins, lamins, elastins, fibronectins, and basement membrane zone-associated components (3). Additionally, increased levels of MMP-2 have been associated with the presence of Porphyromonas endodontalis and P. gingivalis, which are commonly found in primary infection $(32,33)$, as analyzed in the present study.

The linear regression analysis has revealed a positive correlation of MMP-1 with MMP-2 (beta $=0.56, R^{2}=0.952, P<.001$ ) and MMP-9 (beta $=0.57, R^{2}=0.889, P<.001$ ), thus elucidating their association with apical periodontitis. Based on the $R^{2}$ values from this analysis, it is not unreasonable to assume that MMP-1 had a significant implication for the production of MMP-2 and MMP-9 in $95.2 \%$ and $88.9 \%$ of the cases, respectively. Of a clinical relevance, higher levels of MMP1, -2, and -9 were found in teeth with a larger area of bone destruction. Such a finding is in accordance with the literature regarding the assumption that MMPs are involved in jaw cyst expansion and larger areas of bone destruction $(34,35)$. Indeed, Delaissé et al (35) reported that MMP-9 is a cooperative enzyme that participates in the activationresorption and formation cycle involved in both bone remodeling and pathological bone destruction.

In this study, the multivariate logistic regression model elucidated the association between MMPs and the presence of clinical features (13, 15, 16). Higher levels of MMP-2 decreased the chance of clinical symptoms, whereas higher levels of MMP-9 increased it. It is worth pointing out that the gelatinases (MM-2 and -9) appear to regulate neuropathic pain after peripheral nerve injury through degenerative and proinflammatory mechanisms (36). Additionally, MMP-9 expression also appears to mediate pain behavior $(15,37)$. In relation to tenderness to percussion, higher levels of MMP-1 reduced the chance of this outcome, whereas higher levels of MMP-2 increased it. Thus, higher levels of MMP-9 increased the chance of pain on palpation. It should be also highlighted that both tenderness to percussion and pain on palpation are clinical parameters indicative of an inflammatory disease in the periapical tissues.

It is known that MMPs are specifically inhibited by their intrinsic inhibitors, the so-called tissue inhibitors of MMP (TIMPs) (20). TMP-1 and -2 are well characterized and play a multifunctional role as proteins (23). In this study, TIMP-1 and -2 were found in all apical periodontitis, reinforcing their role in the pathogenesis of inflammatory periapical 
lesions $(16,22)$. This result corroborates previous findings showing that the expression of TIMP-1 was also significantly increased in the chronic stages of such lesions $(11,22)$.

Although the MMP's biological activities are independent of the MMP-inhibitory activity, the imbalance between the levels of MMPs and TIMPs is critical for ECM remodeling being able to provoke tissue destruction $(18,23)$. In this study, the linear regression analysis revealed a correlation of MMP-1 with both TIMP-1 (beta $=0.78$, $R^{2}=0.212, P=.041$ ) and TIMP-2 (beta $=1.83, R^{2}=0.231$, $P=.032$ ), which elucidates their association in apical periodontitis. It is worth pointing out that active MMPs are rapidly sequestered by TIMPs (38), and the enzyme-inhibitor complexes cannot be reactivated to any extent. TIMPs also bind to pro-MMPs and regulate MMP activation (ie, MMP-2, membrane type-1 [MT1]-MMP, and TIMP-2 complexes are required for activation of pro-MMP-2) (26). Detailed kinetic studies of the TIMP-MMP associations are reported in the literature (39).

Rather than calculating the ratio of MMPs and TIMPs, we sought to evaluate the formation of MMP/TIMP complexes. To the best of our knowledge, this is the first study investigating the bound form of MMP and TIMP in apical periodontitis. The search for the MMP/ TIMP complex has been speculated by other research fields as a potential biological biomarker for the possible prediction of changes in the course of diseases $(26,27)$. In this study, MMP/TIMP complexes were detected in all apical periodontitis samples, with MMP-9/ TIMP-1 being found at higher levels. No correlation was found in the MMP/TIMP complexes, with no clinical/radiographic feature indicating low or no value in such prediction. Furthermore, future studies should consider the MMP/TIMP complex measurements when comparing acute and chronic infections.

Because bacteria and their by-products (eg, endotoxins) are considered potential modulators of MMP and TIMP expression, we determined the levels of endotoxins present in root canal infection and correlated them with the levels of MMPs and TIMPs found in apical lesions. Endotoxins (ie, LPSs) were detected in all root canal samples from primarily infected root canals $(31,32)$. Higher levels of endotoxins present in root canals were positively correlated with larger amounts of MMP-9. Jotwani et al (40) revealed that LPSs are a relatively potent inducer of MMP-9.

Our findings have revealed that MMPs, TIMPs, and their complexes (MMP/TIMP) are involved in apical periodontitis by interacting with complex networks in the development of clinical features and the severity of bone destruction.

\section{Acknowledgments}

Supported by the Brazilian agencies FAPESP (2015/03697-8; 201219536-5) and CNPq (150557/2011-6).

The authors deny any conflicts of interest related to this study.

\section{References}

1. Nair PN. Pathogenesis of apical periodontitis and the causes of endodontic failures. Crit Rev Oral Biol Med 2004;15:348-81.

2. Tjaderhane L, Hotakainen T, Kinnuen S, et al. The effect of chemical inhibition of matrix metalloproteinases on the size of experimentally induced apical periodontitis. Int Endod J 2007;40:282-9.

3. Buzoglu HD, Unal H, Ulger $\mathrm{C}$, et al. The zymographic evaluation of gelatinase (MMP-2 and -9) levels in acute and chronic periapical abscesses. Oral Surg Oral Med Oral Pathol Oral Radiol Endod 2009;108:121-6.

4. Broverman RL, Nguyen KH, da Silveira A, et al. Changes in the expression of extracellular matrix (ECM) and matrix metalloproteinases (MMP) of proliferating rat parotid acinar cells. J Dent Res 1998;77:1504-14.
5. Hill PA, Murphy G, Docherty AJ, et al. The effects of selective inhibitors of matrix metalloproteinases (MMPs) on bone resorption and the identification of MMPs and TIMP-1 in isolated osteoclasts. J Cell Sci 1994;107:3055-64.

6. Mott JD, Werb Z. Regulation of matrix biology by matrix metalloproteinases. Curr Opin Cell Biol 2004; 16:558-64.

7. Nakopoulou L, Gakiopoulou H, Zervas A, et al. MMP-3 mRNA and MMP-3 and MMP-1 proteins in bladder cancer: a comparison with clinicopathologic features and survival. Appl Immunohistochem Mol Morphol 2001;9:130-7.

8. Vasala K, Pääkkö P, Turpeenniemi-Hujanen T. Matrix metalloproteinase-2 immunoreactive protein as a prognostic marker in bladder cancer. Urology 2003;62:952-7.

9. Guan KP, Ye HY, Yan Z, et al. Serum levels of endostatin and matrix metalloproteinase-9 associated with high stage and grade primary transitional cell carcinoma of the bladder. Urology 2003;61:719-23.

10. Shin SJ, Lee JI, Baek SH, Lim SS. Tissue levels of matrix metalloproteinases in pulps and periapical lesions. J Endod 2002;28:313-5.

11. Paula-Silva FW, da Silva LA, Kapila YL. Matrix metalloproteinase expression in teeth with apical periodontitis is differentially modulated by the modality of root canal treatment. J Endod 2010:36:231-7.

12. Matsui H, Yamasaki M, Nakata K, et al. Expression of MMP-8 and MMP-13 in the development of periradicular lesions. Int Endod J 2011;44:739-45.

13. Shin SJ, Lee W, Lee JI, et al. Matrix metalloproteinase- 8 and substance P levels in gingival crevicular fluid during endodontic treatment of painful, nonvital teeth. Oral Surg Oral Med Oral Pathol Oral Radiol Endod 2011;112:548-54.

14. Menezes-Silva R, Khaliq S, Deeley K, et al. Genetic susceptibility to periapical disease: conditional contribution of MMP2 and MMP3 genes to the development of periapical lesions and healing response. J Endod 2012;38:604-7.

15. Ahmed GM, El-Baz AA, Hashem AA, Shalaan AK. Expression levels of matrix metalloproteinase-9 and gram-negative bacteria in symptomatic and asymptomatic periapical lesions. J Endod 2013;39:444-8.

16. Letra A, Ghaneh G, Zhao M, et al. MMP-7 and TIMP-1, new targets in predicting poor wound healing in apical periodontitis. J Endod 2013;39:1141-6.

17. Beidler SK, Douillet CD, Berndt DF, et al. Multiplexed analysis of matrix metalloproteinases in leg ulcer tissue of patients with chronic venous insufficiency before and after compression therapy. Wound Repair Regen 2008;16:642-8.

18. Liu Y, Min D, Bolton T, et al. Increased matrix metalloproteinase-9 predicts poor wound healing in diabetic foot ulcers. Diabetes Care 2009;32:117-9.

19. Lu L, Gunja-Smith Z, Woessner JF, et al. Matrix metalloproteinases and collagen ultrastructure in moderate myocardial ischemia and reperfusion in vivo. Am J Physiol Heart Circ Physiol 2000;279:601-9.

20. Edwards DR, Beaudry PP, Laing TD, et al. The roles of tissue inhibitors of metalloproteinases in tissue remodelling and cell growth. Int J Obes Relat Metab Disord 1996;20:9-15.

21. Murate T, Hayakawa T. Multiple functions of tissue inhibitors of metalloproteinases (TIMPs): new aspects in hematopoiesis. Platelets 1999;10:5-16.

22. Garlet GP, Horwat R, Ray HL Jr, et al. Expression analysis of wound healing genes in human periapical granulomas of progressive and stable nature. J Endod 2012;38: 185-90.

23. Nagase H, Woessner JF Jr. Matrix metalloproteinases. J Biol Chem 1999;274:21491-4.

24. Sobue T, Hakeda Y, Kobayashi Y, et al. Tissue inhibitor of metalloproteinases 1 and 2 directly stimulate the bone-resorbing activity of isolated mature osteoclasts. J Bone Miner Res 2001;16:2205-14.

25. Docherty AJ, Lyons A, Smith BJ, et al. Sequence of human tissue inhibitor of metalloproteinases and its identity to erythroid-potentiating activity. Nature 1985;318: 66-9.

26. Shen Q, Lee ES, Pitts RL, et al. Tissue inhibitor of metalloproteinase-2 regulates matrix metalloproteinase-2-mediated endothelial barrier dysfunction and breast cancer cell transmigration through lung microvascular endothelial cells. Mol Cancer Res 2010;8:939-51.

27. Thorsen SB, Christensen SL, Würtz SO, et al. Plasma levels of the MMP-9: TIMP-1 complex as prognostic biomarker in breast cancer: a retrospective study. BMC Cancer 2013;13:598.

28. Anne TM, Taina TH. Levels of circulating TIMP-2 and MMP2-TIMP2 complex are decreased in squamous cervical carcinoma. Obstet Gynecol Int 2010;179351.

29. Pennanen H, Kuittinen O, Turpeenniemi-Hujanen T. Plasma MMP-2-TIMP-2 complex levels measured during follow-up predict a risk of relapse in patients with malignant lymphoma. Eur J Haematol 2008;80:46-54.

30. Vasala K, Turpeenniemi-Hujanen T. Serum tissue inhibitor of metalloproteinase-2 (TIMP-2) and matrix metalloproteinase-2 in complex with the inhibitor (MMP2:TIMP-2) as prognostic markers in bladder cancer. Clin Biochem 2007:40:640-4.

31. Martinho FC, Nascimento GG, Leite FR, et al. Clinical influence of different intracanal medications on Th1-type and Th2-type cytokine responses in apical periodontitis. J Endod 2015;41:169-75.

32. Martinho FC, Leite FR, Chiesa WM, et al. Signaling pathways activation by primary endodontic infectious contents and production of inflammatory mediators. J Endod 2014;40:484-9. 


\section{Basic Research-Biology}

33. Siqueira JF Jr, Rôças IN, Silva MG. Prevalence and clonal analysis of Porphyromonas gingivalis in primary endodontic infections. J Endod 2008;34:1332-6.

34. Carneiro E, Menezes R, Garlet GP, et al. Expression analysis of matrix metalloproteinase- 9 in epithelialized and nonepithelialized apical periodontitis lesions. Oral Surg Oral Med Oral Pathol Oral Radiol Endod 2009;107:127-32.

35. Delaissé JM, Ledent P, Vaes G. Collagenolytic cysteine proteinases of bone tissue. Cathepsin B, (pro)cathepsin L and a cathepsin L-like $70 \mathrm{kDa}$ proteinase. Biochem J 1991;279:167-74.

36. Kobayashi H, Chattopadhyay S, Kato K, et al. MMPs initiate Schwann cell-mediated MBP degradation and mechanical nociception after nerve damage. Mol Cell Neurosci 2008;39:619-27.
37. Ji RR, Xu ZZ, Wang X, Lo EH. Matrix metalloprotease regulation of neuropathic pain. Trends Pharmacol Sci 2009;30:336-40.

38. Cawston TE, Murphy G, Mercer E, et al. The interaction of purified rabbit bone collagenase with purified rabbit bone metalloproteinase inhibitor. Biochem J 1983;211: 313-8.

39. Hutton M, Willenbrock F, Brocklehurst K, Murphy G. Kinetic analysis of the mechanism of interaction of full-length TIMP-2 and gelatinase A: evidence for the existence of a low-affinity intermediate. Biochemistry 1998;37:10094-8.

40. Jotwani R, Eswaran SV, Moonga S, Cutler CW. MMP-9/TIMP-1 imbalance induced in human dendritic cells by Porphyromonas gingivalis. FEMS Immunol Med Microbiol 2010;58:314-21. 